\title{
Liste des thèses d'anthropologie soutenues en 2016
}

\section{Université d'Aix-Marseille}

Federico Carlini : Étude de la dynamique de l'expression de la molécule $H L A-G$, en contexte sain et pathologique, en fonction du génotype HLA et de facteurs externes.

Louise Corron: Juvenile age estimation in physical anthropology: a critical review of existing methods and the application of two standardised methodological approaches.

Lætitia Lanteri: Recrutement, paléodémographie et cémentochronologie. Application à un contexte d'inhumation paroissial d'Ancien Régime: Notre-Dame-du-Bourg de Digne.

\section{Université de Bordeaux}

Nada Helias: Pratiques funéraires et paramètres biologiques au Levant romain: étude de la nécropole de Berythus (Liban).

Sacha Kacki : Influence de l'état sanitaire des populations anciennes sur la mortalité en temps de peste.

Mona Le Luyer: Évolution de l'architecture interne des dents humaines à la fin du Pléistocène et au début de l'Holocène.

Thi Thuy Nga Nguyen: Étude tridimensionnelle de la forme et de la position de l'os hyoide, sa relation avec les voies aériennes supérieures et d'autres structures cervicofaciales. Comparaison entre populations et perspectives anthropologiques.

Maïté Rivollat: Diversité génétique de la population néo-inhumée à Gurgy (Yonne). Apport de l'approche paléogénétique à la compréhension du fonctionnement du site et des processus de Néolithisation du continent européen.

\section{Université de Poitiers}

Ghislain Thiery: Analyse morpho-fonctionnelle de la topographie dentaire $3 D$ chez les primates actuels et fossiles.

\section{Université de Toulouse-III}

Manon-Anne Barutaut : Découverte et validation de nouveaux biomarqueurs de l'insuffisance cardiaque.

Caroline Capuani : Lésions osseuses par armes blanches : analyse des caractéristiques lésionnelles et détermination du mécanisme de production en macroscopie à épifluorescence.

Marwan El Khoury : Variabilité anatomique des canaux semi-circulaires chez Homo sapiens, Pan paniscus et Pan troglodytes en morphométrie $3 D$.

Lei Pan : The earliest members of genus Homo in South Africa: evidence from inner structure of lower postcanine dentition.

\section{Muséum national d'Histoire naturelle}

Kisito Essele Essele : Continuités et innovations sonores des cérémonies funéraires des Éton du Sud Cameroun.

Sandrine Gallois : Dynamiques des savoirs écologiques locaux chez les enfants Baka du Sud-Est Cameroun.

Julie Landes : Compromis entre traits d'histoire de vie et saisonnalité chez un primate hétérotherme.

Pauline Laval : Captures estuariennes, une ethnoécologie de la pêche sur le bas Oyapock (frontière franco-brésilienne).

Julie Scapino: De la friche urbaine à la biodiversité. Ethnologie d'une reconquête (la petite ceinture de Paris). 